\title{
On Second Riesz $\Phi$-Variation of Normed Space Valued Maps
}

\author{
Mireya Bracamonte ${ }^{1}$, José Giménez ${ }^{2}$, N. Merentes $^{3}$, J. L. Sánchez ${ }^{3}$ \\ ${ }^{1}$ Departamento de Matemáticas, Universidad Centroccidental Lisandro Alvarado, Barquisimeto, Venezuela \\ ${ }^{2}$ Departamento de Matemáticas, Universidad de los Andes, Mérida, Venezuela \\ ${ }^{3}$ Departamento de Matemáticas, Universidad Central de Venezuela, Caracas, Venezuela \\ Email: mireyabracamonte@ucla.edu.ve,jgimenez@ula.ve,nmerucv@gmail.com,jose.sanchez@ciens.ucv.ve
}

Received September 21, 2011; revised November 11, 2011; accepted November 20, 2011

\begin{abstract}
In this article we present a Riesz-type generalization of the concept of second variation of normed space valued functions defined on an interval $[a, b] \subset \mathbb{R}$. We show that a function $f \in X^{[a, b]}$, where $X$ is a reflexive Banach space, is of bounded second $\Phi$-variation, in the sense of Riesz, if and only if it can be expressed as the (Bochner) integral of a function of bounded (first) $\$$ Phi \$-variation. We provide also a Riesz lemma type inequality to estimate the total second Riesz- $\Phi$-variation introduced.
\end{abstract}

Keywords: Young Function; $\Phi$-Variation; Second $\Phi$-Variation of a Function

\section{Introduction}

Functions of bounded variation where first introduced in 1881 by Camille Jordan who established the relation between these functions and the monotonic ones. Thus, the Dirichlet Criterion for the convergence of the Fourier series applies to the class of functions of bounded variation. This, in turn, has motivated the study of solutions of nonlinear equations that describe concrete physical phenomena in which, often, functions of bounded variation intervene.

The interest generated by this notion has lead to some generalizations of the concept, mainly, intended to the search of a bigger class of functions whose elements have point wise convergent Fourier series. As in the classical case, these generalizations have found many applications in the study of certain differential and integral equations. Ch. J. de la Vallée Poussin, introduced in 1908 ([1]) the notion of second variation of a function. A few years later, F. Riesz ([2]) proved that a function $f$ is of bounded second variation on $[a, b]$ if, and only if, it is the definite Lebesgue integral of a function $F$ of bounded variation.

More recently, in 1983, A. M. Russell and C. J. F. Upton [4] obtained a similar result for functions of bounded second variation $(\$ 1 \leq p \leq \infty)$, in the sense of Wiener. In 1992 the third author introduced the notion of $(p, 2)$ variation, in the sense of Riesz ([4]), presenting, also, a result that generalizes the renowned Riesz lemma for the class that he called $B V_{p}^{2}([a, b])$, or class of functions of bounded Riesz $(p, 2)$-variation.

In this article we define the notion of function of bounded second $\Phi$-variation in the sense of Riesz. We show that a function $F$, with values in a reflexive Banach space, is of bounded second $\Phi$-variation, in the sense of Riesz, if and only if it is the integral (in the sense of Bochner) of a function of bounded $\Phi$-variation. In addition, from the main results presented it is deduced an inequality that generalizes Riesz's lemma.

\section{Vector Value Functions of Bounded Variation}

We begin this section by recalling some known spaces and results.

We will also assume that all partitions of an interval $[a, b]$ considered, contain at least one point $t \in(a, b)$; the set of all such partitions will be denoted as $\pi_{3}[a, b]$.

The notion of bounded second variation in the sense De La Vallée Poussin is defined as follows: A function $u:[a, b] \rightarrow \mathbb{R}$ is of bounded second variation if and only if

$$
V^{2}(u ;[a, b]):=\sup _{\pi \in \Pi_{3}([a, b])} \sum_{i=0}^{m-2}\left|u\left[t_{i+1}, t_{i+2}\right]-u\left[t_{i}, t_{i+1}\right]\right|<\infty
$$

where

$$
u\left[t_{i+1}, t_{i+2}\right]:=\frac{u\left(t_{i+2}\right)-u\left(t_{i+1}\right)}{t_{i+2}-t_{i+1}} \text { and } \pi=\left\{t_{0}, t_{2}, \cdots, t_{m}\right\} .
$$


The class of all the functions of bounded second variation (on $[a, b]$ ), in the sense of De La Vallée Poussin, is denoted by $B V^{2}([a, b])$.

The following are known properties of functions in $B V^{2}([a, b])([3,5,6])$.

Proposition 2.1. Let $u \in B V^{2}([a, b])$. then

1) If $v \in B V^{2}([a, b])$ and $\lambda$ is any real constant,

$$
\begin{gathered}
V^{2}(\lambda u ;[a, b])=|\lambda| V^{2}(u ;[a, b]) \\
y \\
V^{2}(u+v ;[a, b]) \leq V^{2}(u ;[a, b])+V^{2}(v ;[a, b]) .
\end{gathered}
$$

2) (Monotonicity) If $a<c<d<b$ then $V^{2}(u ;[c, d]) \leq V^{2}(u ;[a, b])$.

3) (Semi-additive) $a<c<b$, then $u \in B V^{2}([a, c])$, $u \in B V^{2}([c, b])$ and

$$
V^{2}(u ;[a, b]) \geq V^{2}(u ;[a, c])+V^{2}(u ;[c, b]) .
$$

4) $u\left[y_{0}, y_{1}\right]$ is bounded for all $y_{0}, y_{1} \in[a, b]$.

5) $u$ is Lipschitz and therefore absolutely continuous on $[a, b]$.

6) $u \in B V^{2}([a, b])$ if and only if $u=u_{1}-u_{2}$, where $u_{1}, u_{2}$ are convex functions.

7) A necessary and sufficient condition for a function $F$ to be the integral of a function $f \in B V([a, b])$ is that $F \in B V^{2}([a, b])$. This result is known as Riesz's lemma.

8) If $u$ is twice differentiable with $u^{\prime \prime}$ integrable on $[a, b]$ then $u \in B V^{2}([a, b])$ and

$$
V^{2}(u ;[a, b])=\int_{a}^{b}\left|u^{\prime \prime}(t)\right| \mathrm{d} t .
$$

In 1910, F. Riesz introduced the concept of function of bounded $p$-variation $(1<p<\infty)$ as follows:

Definition 2.2. Let $1 \leq p<\infty$. A function

$f:[a, b] \subset \mathbb{R} \rightarrow \mathbb{R}$ is said to be of bounded p-variation, in the sense of Riesz, if

$$
\begin{aligned}
V_{p}^{R}(f) & =V_{p}^{R}(f ;[a, b]) \\
& :=\sup _{\pi \in \Pi_{3}([a, b])} \sum_{i=0}^{m-2}\left|u\left[t_{i}, t_{i+1}\right]\right|^{p}\left(t_{i+1}-t_{i}\right)<\infty .
\end{aligned}
$$

The class of all functions of bounded $p$-variation on $[a, b]$, in the sense of Riesz, is denoted by $R B V_{p}[a, b]$.

It readily follows from the definitions that $R B V_{p}[a, b] \subset C[a, b]$ and that for all $f, g \in R B V_{p}[a, b]$

$$
V_{p}^{R}(f g) \leq\left\|f^{p}\right\|_{\infty} V_{p}^{R}(g)+\left\|g^{p}\right\|_{\infty} V_{p}^{R}(f) ;
$$

in fact, the relation $\|f\|_{p}:=|f(a)|+\left(V_{p}^{R}(f)\right)^{\frac{1}{p}}$, defines a norm in $R B V_{p}[a, b]$ respect to which it becomes a Banach algebra (see e.g., [7]).

Notice that $R B V_{1}[a, b]=B V[a, b]$; on the other hand, it is well known that, for $1<p<\infty$ a function belongs to $R B V_{p}[a, b]$ if, and only if, it is absolutely continuous and its derivative (which exists $\lambda$-a.e. in $[a, b]$ ) belongs to $L_{p}[a, b]$; in this case $V_{p}^{R}(f ;[a, b])=\left\|f^{\prime}\right\|_{L_{p}}^{p}$ (this is the renowned Riesz's lemma, [2]). In particular, $\operatorname{Lip}[a, b] \subset R B V_{p}[a, b]$.

Further generalizations consider the so call $\varphi$-functions. As it is customary, we shall denote by $\mathcal{N}$ the set of all continuous convex functions $\varphi:[0,+\infty) \rightarrow[0,+\infty)$ such that $\varphi(\rho)=0$ if and only if $\rho=0$ and $\lim _{\rho \rightarrow 0^{+}} \frac{\varphi(\rho)}{\rho}=0$. Likewise, the notation $\mathcal{N}_{\infty}$ shall be used to denote the set of all functions $\varphi \in \mathcal{N}$, for which the Orlicz condition holds: $\lim _{\rho \rightarrow \infty} \frac{\varphi(\rho)}{\rho}=+\infty$. Following [8] functions in $\mathcal{N}$ shall be called $\varphi$-functions. Any function $\varphi \in \mathcal{N}$ is strictly increasing, and the function $\rho \mapsto \frac{\varphi(\rho)}{\rho}$ is no decreasing for $\rho>0$.

One says that a function $\Phi \in \mathcal{N}$ satisfies a condition $\Delta_{2}$, and writes $\Phi \in \Delta_{2}$, if there are constants $K>2$ and $t_{0} \in \mathbb{R}$ such that

$$
\Phi(2 t) \leq K \Phi(t) \text { for all } t \geq t_{0} .
$$

For instance, if $\Phi(x):=t^{p}, p>1$, one may chooses the optimal constant $K=2^{p}$.

In the sequel we will assume that $\Phi$ is a $\varphi$-function and $X$ is a normed space with norm $\|\cdot\|_{X}$ (or simply $\|\cdot\|$ ). The integral of a normed space valued function means the Bochner integral ([9]).

The following generalization of the notion of function of bounded variation is due to V. V. Chistyakov ([11], see also [12]).

Definition 2.3. The $\Phi$-variation in sense of Riesz of a map $f:[a, b] \rightarrow X$ is defined as

$$
\begin{aligned}
V_{\Phi}^{R}(f,[a, b]) & =V_{\Phi}^{R}(f,[a, b], X) \\
& :=\sup _{\xi} \sum_{i=1}^{n} \Phi\left(\frac{\left\|f\left(t_{i}\right)-f\left(t_{i-1}\right)\right\|}{t_{i}-t_{i-1}}\right)\left(t_{i}-t_{i-1}\right)
\end{aligned}
$$

where the supremun is taking over the set of all partitions $\xi=\left\{t_{i}\right\}_{i=0}^{n}$ of the interval $[a, b]$.

Some known properties of the functional $V_{\Phi}^{R}$ are the following

1) $V_{\Phi}^{R}$ is no decreasing, that is, $V_{\Phi}^{R}(f,[a, b]) \leq V_{\Phi}^{R}(f,[c, d])$ if $[a, b] \subset[c, d]$;

2) $V_{\Phi}^{R}$ is semi additive

$V_{\Phi}^{R}(f,[a, c])+V_{\Phi}^{R}(f,[c, b]) \leq V_{\Phi}^{R}(f,[a, b])$ for all

$a<c<b$; and

3) $V_{\Phi}^{R}$ is sequentially lower semi continuous.

The set of all functions $f \in X^{[a, b]}$ for which $V_{\Phi}^{R}(f,[a, b])<\infty$ is not necessarily a linear space, but 
it is a convex subset of $X^{[a, b]}$ and $V_{\Phi}^{R}(\cdot,[a, b])$ is a convex functional on it.

The class

$$
B_{\Phi}^{R}([a, b], X):=\left\{f \in X^{[a, b]}: \exists \lambda>0, V_{\Phi}^{R}(f / \lambda)<\infty\right\}
$$

is a linear space, called the class of functions of bounded $\Phi$-variation, in the sense of Riesz. It can be equipped with the norm: $\|f\|:=\|f(a)\|+\rho_{\Phi}(f)$, where

$$
\rho_{\Phi}(f):=\inf \left\{\lambda>0: V_{\Phi}(f \lambda)<1\right\} .
$$

Definition 2.4. ([11]) A mapping $f:[a, b] \rightarrow X$ is called absolutely continuous if there exists a function $\delta:(0, \infty) \rightarrow(0, \infty)$ such that for any $\epsilon>0, n \in \mathbb{N}$ and any finite collection of points $\left\{a_{i}, b_{i}\right\}_{i=1}^{n} \subset[a, b]$ such that $a_{1}<b_{1} \leq a_{2}<b_{2} \leq \cdots \leq a_{n}<b_{n}$, the condition

$\sum_{i=1}^{n}\left(b_{i}-a_{i}\right)<\delta(\epsilon)$ implies $\sum_{i=1}^{n}\left\|f\left(b_{i}\right)-f\left(a_{i}\right)\right\|<\epsilon$.

A proof of the following result can be found in [12].

Proposition 2.5. Let $X$ be a reflexive Banach space. Then every $X$-valued absolutely continuous function $f$, defined on $[a, b]$, is a.e. strongly differentiable and can be represented as

$$
f(t)=f(a)+\int_{a}^{t} f^{\prime}(s) \mathrm{d} s, t \in[a, b],
$$

where $f^{\prime}$ denotes the strong derivative of $f$.
We now state Theorem 3.3 of [11].

Theorem 2.6. Let $(X,\|\| \|)$ be a reflexive Banach space. Suppose that $\Phi \in \mathcal{N}$ and that $f \in B V_{\Phi}([a, b] ; X)$.

Then $f$ admits a strong derivative $f^{\prime}(t) \in X$ for almost all $t \in[a, b]$ which is strongly measurable and $\int_{a}^{b} \Phi\left(\left\|f^{\prime}(t)\right\|\right) \mathrm{d} t \leq V_{\Phi}(f,[a, b])$. Moreover, if $\Phi \in \mathcal{N}_{\infty}$, then $f$ absolutely continuous and the following integral formula for the variation holds:

$$
f V_{\Phi}^{R}(f,[a, b])=\int_{a}^{b} \Phi\left(\left\|f^{\prime}(t)\right\|\right) \mathrm{d} t .
$$

Now we introduce a notion that generalizes the classical de la Vallée Poussin's concept of second bounded variation.

Definition 2.7. Given $f:[a, b] \rightarrow X$, $\xi=\left\{x_{j}\right\}_{j=0}^{m} \in \pi_{3}[a, b]$ and $\Phi \in \mathcal{N}$. We shall use the following notation:

$$
U\left(f ; x_{j}, x_{i}\right):=\frac{f\left(x_{i}\right)-f\left(x_{j}\right)}{x_{i}-x_{j}}
$$

and define the second variation of a function $f$ on $[a, b]$, in the sense of Riesz, as

$$
V_{\Phi}^{2, R}(f ;[a, b], X):=\sup _{\xi \in \pi_{3}[a, b]} \varrho_{\Phi}^{2}(f ;[a, b], \xi)
$$

where

$$
\varrho_{\Phi}^{2}(f ;[a, b], \xi):=\sum_{j=0}^{m-2} \Phi\left(\frac{\left\|U\left(f ; x_{j+1}, x_{j+2}\right)-U\left(f ; x_{j}, x_{j+1}\right)\right\|}{x_{j+2}-x_{j}}\right)\left(x_{j+2}-x_{j}\right) .
$$

If $V_{\Phi}^{2, R}(f ;[a, b], X)<\infty$ we will say that the function $f$ is of bounded second $\Phi$-variation, in the sense of Riesz, and write $f \in B V_{\Phi}^{2, R}([a, b], X)$.

Lemma 2.8. Let $f \in B V_{\Phi}^{2, R}([a, b], X)$. Then

1) For $x<y<z$ in $[a, b]$ there is a constant $K>0$ such that $\|U(f ; z, y)-U(f ; y, x)\| \leq K$,
2) $U\left(f ; y_{0}, y_{1}\right)$ is bounded for all $y_{0}, y_{1} \in[a, b]$,

3) $f$ is absolutely continuous on $[a, b]$,

4) If $x_{0}, y_{0} \in[a, b]$ with $x_{0} \neq y_{0}$ then $U\left(f ; x_{0}, x\right)$ is continuous at $x=y_{0}$.

Proof. 1) Consider three points $x<y<z$ in $[a, b]$, and note that if $z-x \leq 1$.

$$
\begin{aligned}
\|U(f ; z, y)-U(f ; y, x)\| & =\Phi^{-1}(\Phi(\|U(f ; z, y)-U(f ; y, x)\|))=\Phi^{-1}\left(\Phi\left((z-x) \frac{\|U(f ; z, y)-U(f ; y, x)\|)}{z-x}\right)\right) \\
& \leq \Phi^{-1}\left(\Phi\left(\frac{\|U(f ; z, y)-U(f ; y, x)\|)}{z-x}\right)(z-x)\right) \leq \Phi^{-1}\left(V_{\Phi}^{2, R}(f ;[a, b], X)\right) .
\end{aligned}
$$

If instead $z-x>1$

$$
\begin{aligned}
\|U(f ; z, y)-U(f ; y, x)\| & =\frac{\|U(f ; z, y)-U(f ; y, x)\|}{z-x}(z-x)=\Phi^{-1}\left(\Phi\left(\frac{\|U(f ; z, y)-U(f ; y, x)\|)}{z-x}\right)(z-x)\right. \\
& =\Phi^{-1}\left(\Phi\left(\frac{\|U(f ; z, y)-U(f ; y, x)\|}{z-x}\right)(z-x) \frac{1}{z-x}\right)(z-x) \\
& \leq \Phi^{-1}\left(V_{\Phi}^{2, R}(f ;[a, b], X) \frac{1}{z-x}\right)(z-x) \leq \Phi^{-1}\left(V_{\Phi}^{2, R}(f ;[a, b], X)\right)(b-a) .
\end{aligned}
$$


Then $\| U(f ; z, y))-U(f ; y, x) \| \leq K$ where

$$
\begin{gathered}
K:=\max \left\{\Phi^{-1}\left(V_{\Phi}^{2, R}(f ;[a, b], X)\right)(b-a),\right. \\
\left.\Phi^{-1}\left(V_{\Phi}^{2, R}(f ;[a, b], X)\right)\right\} .
\end{gathered}
$$

2) Let $y_{0}, y_{1} \in[a, b]$ and let $c \in(a, b)$. The proof depends on the location of $y_{0}, y_{1}$ with respect to $a, b$ and $c$.

Case 1: $a<y_{0}<c<y_{1}<b$. In this case, for $y_{2} \in\left(y_{1}, b\right)$, we have

$$
\begin{aligned}
\left\|U\left(f ; y_{0}, y_{1}\right)\right\| \leq & \left\|U\left(f ; y_{0}, y_{1}\right)-U\left(f ; y_{1}, y_{2}\right)\right\|+\left\|U\left(f ; y_{1}, y_{2}\right)-U\left(f ; c, y_{1}\right)\right\| \\
& +\left\|U\left(f ; c, y_{1}\right)-U(f ; a, c)\right\|+\|U(f ; a, c)\| \\
\leq & 3 K+\|U(f ; a, c)\| .
\end{aligned}
$$

where $K$ is given by 1 ).

Case 2: $a<y_{-} 0<c<y_{-} 1=b$. Here, for $y_{2} \in\left(c, y_{1}\right)$

$$
\begin{aligned}
\left\|U\left(f ; y_{-} 0, y_{-} 1\right)\right\| & \leq\left\|U\left(f ; y_{0}, y_{1}\right)-U\left(f ; a, y_{0}\right)\right\|+\left\|U\left(f ; a, y_{0}\right)-U\left(f ; y_{0}, y_{2}\right)\right\| \\
& +\left\|U\left(f ; y_{0}, y_{2}\right)-U\left(f ; y_{1}, y_{2}\right)\right\|+\left\|U\left(f ; y_{1}, y_{2}\right)-U\left(f ; c, y_{2}\right)\right\| \\
& +\left\|U\left(f ; c, y_{2}\right)-U(f ; a, c)\right\|+\|U(f ; a, c)\| \leq K+\|U(f ; a, c)\| .
\end{aligned}
$$

where $K$ is given by 1 ). In any other case one proceeds in a similar fashion.

Since $c$ is arbitrary but fixed $U\left(f ; y_{0}, y_{1}\right)$ \#must be bounded.

3) By 2) there exists $M \geq 0$ such that $\left\|U\left(f ; y_{0}, y_{1}\right)\right\| \leq M$ \#for all $y_{0}, y_{1} \in[a, b]$. Therefore $f$ is Lipschitz, and hence, absolutely continuous.

4) It is immediate.
Ejemplo 2.9. Let $C_{0}$ be the subspace of $\ell_{\infty}$ of all null sequences equipped with the natural norm inherited from $\ell_{\infty}$. Let $f:[a, b] \rightarrow C_{0},\|\cdot\|_{\infty}$ be defined by $f(t):=\left(\frac{|t|}{n}\right)_{n \in \mathbb{N}}$. If $a>0$, then for every partition $\xi=\left\{x_{i}\right\}_{j=0}^{m} \in \pi_{3}[a, b]$ :

$$
\begin{aligned}
& \sum_{j=0}^{m-2} \Phi\left(\frac{\left\|\frac{f\left(x_{j+2}\right)-f\left(x_{j+1}\right)}{x_{j+2}-x_{j+1}}-\frac{f\left(x_{j+1}\right)-f\left(x_{j}\right)}{x_{j+1}-x_{j}}\right\|_{\infty}}{x_{j+2}-x_{j}}\right)\left(x_{j+2}-x_{j}\right) \\
& =\sum_{j=0}^{m-2} \Phi\left(\frac{\left\|\frac{\left(\frac{x_{j+2}}{n}\right)_{n \in \mathbb{N}}-\left(\frac{x_{j+1}}{n}\right)_{n \in \mathbb{N}}}{x_{j+2}-x_{j+1}}-\frac{\left(\frac{x_{j+1}}{n}\right)_{n \in \mathbb{N}}-\left(\frac{x_{j}}{n}\right)_{n \in \mathbb{N}}}{x_{j+1}-x_{j}}\right\|}{x_{j+2}-x_{j}} \|_{\infty}\right)\left(x_{j+2}-x_{j}\right)=\sum_{j=0}^{m-2} \Phi\left(\frac{\left\|(0)_{n \in \mathbb{N}}\right\|}{x_{j+2}-x_{j}}\right)\left(x_{j+2}-x_{j}\right)=0,
\end{aligned}
$$

which means $f \in B V_{\Phi}^{2, R}([a, b], X)$ and $V_{\Phi}^{2, R}(f ;[a, b], X)=0$. A similar estimation holds if $a<b<0$.

On the other hand, consider the same function

$$
\begin{aligned}
& f(t):=\left(\frac{|t|}{n}\right)_{n \in \mathbb{N}} \text { on the interval }\left[-\frac{2}{3}, \frac{2}{3}\right] \text { and let } \\
& \left\{-\frac{2}{3},-\frac{1}{3}, \frac{1}{3}, \frac{2}{3}\right\} \in \pi_{3}\left(\left[-\frac{2}{3}, \frac{2}{3}\right]\right) \text {. Then: }
\end{aligned}
$$




$$
\begin{aligned}
& \Phi\left(\frac{\left\|\frac{f(1 / 3)-f(-1 / 3)}{1 / 3-(-1 / 3)}-\frac{f(-1 / 3)-f(-2 / 3)}{-1 / 3-(-2 / 3)}\right\|_{\infty}}{1 / 3-(-2 / 3)}\right)(1 / 3-(-2 / 3)) \\
& +\Phi\left(\frac{\left\|\frac{f(2 / 3)-f(1 / 3)}{2 / 3-1 / 3}-\frac{f(1 / 3)-f(-1 / 3)}{1 / 3-(-1 / 3)}\right\|_{\infty}}{2 / 3-(-1 / 3)}\right)_{n}(2 / 3-(-1 / 3)) \\
& =\Phi\left(\left\|\frac{\left.\| \frac{\left(\frac{1 / 3}{n}\right)_{n \in \mathbb{N}}-\left(\frac{2 / 3}{n}\right)_{n \in \mathbb{N}} \|}{1 / 3}\right)+\Phi\left(\| \frac{2 / 3}{n}\right)_{n \in \mathbb{N}}-\left(\frac{1 / 3}{n}\right)_{n \in \mathbb{N}} \|}{1 / 3}\right\|\right) \\
& =\Phi\left(\left\|\left(\frac{1}{n}\right)_{n \in \mathbb{N}}\right\|_{\infty}\right)+\Phi\left(\left\|\left(\frac{1}{n}\right)_{n \in \mathbb{N}}\right\|\right)=2 \Phi\left(\left\|\left(\frac{1}{n}\right)_{n \in \mathbb{N}}\right\|_{\infty} \|\right)=2 \Phi(1) .
\end{aligned}
$$

So that, in this case $V_{\Phi}^{2, R}(f ;[-2 / 3,2 / 3]) \geq 2 \Phi(1)>0$.

Lemma 2.10. If $f, g \in B V_{\Phi}^{2, R}([a, b], X), \lambda$ is a complex constant with $|\lambda| \leq 1$ and $\alpha, \beta \in[0,1]$ such that $\alpha+\beta=1$, then

1) $V_{\Phi}^{2, R}(\lambda f ;[a, b], X) \leq|\lambda| V_{\Phi}^{2, R}(f ;[a, b], X) \$$,

2) $V_{\Phi}^{2, R}$ is convex in the function argument; that is

$$
\begin{aligned}
V_{\Phi}^{2, R}(\alpha f+\beta g ;[a, b], X) \leq & \alpha V_{\Phi}^{2, R}(f ;[a, b], X) \\
& +\beta V_{\Phi}^{2, R}(g ;[a, b], X) .
\end{aligned}
$$

We omit the proof, which follows immediately from the convexity of $\Phi$

Lemma 2.11. Let $f \in B V_{\Phi}^{2, R}([a, b], X)$ and $c \in(a, b)$, then $f \in B V_{\Phi}^{2, R}([a, b]) \cap B V_{\Phi}^{2, R}([c, b])$ and

$$
V_{\Phi}^{2, R}(f ;[a, c])+V_{\Phi}^{2, R}(f ;[c, b]) \leq V_{\Phi}^{2, R}(f ;[a, b], X) .
$$

Proof. Let $c \in(a, b)$ and let $\xi=\left\{x_{i}\right\}_{j=0}^{m} \in \pi_{3}[a, c]$. Then, $\xi \cup\{b\} \in \pi_{3}[a, b]$. Hence

$$
\begin{aligned}
& \varrho_{\Phi}^{2}(f ;[a, c], \xi)=\sum_{j=0}^{m-2} \Phi\left(\frac{\left\|\frac{f\left(x_{j+2}\right)-f\left(x_{j+1}\right)}{x_{j+2}-x_{j+1}}-\frac{f\left(x_{j+1}\right)-f\left(x_{j}\right)}{x_{j+1}-x_{j}}\right\|}{x_{j+2}-x_{j}}\right)\left(x_{j+2}-x_{j}\right) \\
& \leq \sum_{j=0}^{m-2} \Phi\left(\frac{\left\|\frac{f\left(x_{j+2}\right)-f\left(x_{j+1}\right)}{x_{j+2}-x_{j+1}}-\frac{f\left(x_{j+1}\right)-f\left(x_{j}\right)}{x_{j+1}-x_{j}}\right\|}{x_{j+2}-x_{j}}\right)\left(x_{j+2}-x_{j}\right)+\Phi\left(\frac{\left\|\frac{f(b)-f\left(x_{m}\right)}{b-x_{m}}-\frac{f\left(x_{m}\right)-f\left(x_{m-1}\right)}{x_{m}-x_{m-1}}\right\|}{b-x_{m-1}}\right) \\
& \leq V_{\Phi}^{2, R}(f ;[a, b], X) .
\end{aligned}
$$

Since this holds for all partitions $\xi=\left\{x_{i}\right\}_{j=0}^{m}$ of $[a, c]$, then $\xi \cup \eta \in \pi_{3}[a, b]$. Put $\tau:=\left\{y_{j}\right\}_{j=0}^{m+n}$, where it follows that $f \in B V_{\Phi}^{2, R}(f ;[a, c], X)$ and $V_{\Phi}^{2, R}(f ;[a, c], X) \leq V_{\Phi}^{2, R}(f ;[a, b], X)$. Similarly, one gets $V_{\Phi}^{2, R}(f ;[c, b], X) \leq V_{\Phi}^{2, R}(f ;[a, b], X)$. On the other

hand, if $\xi=\left\{x_{j}\right\}_{j=0}^{m} \in \pi_{3}[a, c] \quad \eta=\left\{x^{j}\right\}_{j=0}^{n} \in \pi_{3}[c, b], \quad$ Then

$$
y_{j}:=\left\{\begin{array}{lll}
x_{j} & \text { si } & 0 \leq j \leq m \\
x^{j-m} & \text { si } & m \leq j \leq n+m .
\end{array}\right.
$$




$$
\begin{aligned}
& \varrho_{\Phi}^{2}(f ;[a, c], \xi)+\varrho_{\Phi}^{2}(f ;[c, b], \eta) \leq \sum_{j=0}^{m-2} \Phi\left(\frac{\left\|\frac{f\left(x_{j+2}\right)-f\left(x_{j+1}\right)}{x_{j+2}-x_{j+1}}-\frac{f\left(x_{j+1}\right)-f\left(x_{j}\right)}{x_{j+1}-x_{j}}\right\|}{x_{j+2}-x_{j}}\right)\left(x_{j+2}-x_{j}\right) \\
& +\Phi\left(\frac{\left\|\frac{f\left(x^{1}\right)-f\left(x^{0}\right)}{x^{1}-x^{0}}-\frac{f\left(x^{0}\right)-f\left(x_{m-1}\right)}{x^{0}-x_{m-1}}\right\|}{x^{1}-x_{m-1}}\right)\left(x^{1}-x_{m-1}\right)+\sum_{j=0}^{n-2} \Phi\left(\frac{\left\|\frac{f\left(x^{j+2}\right)-f\left(x^{j+1}\right)}{x^{j+2}-x^{j+1}}-\frac{f\left(x^{j+1}\right)-f\left(x^{j}\right)}{x^{j+1}-x^{j}}\right\|}{x^{j+2}-x^{j}}\right)\left(x^{j+2}-x^{j}\right) \\
& =\sum_{j=0}^{m+n-2} \Phi\left(\frac{\left\|\frac{f\left(y_{j+2}\right)-f\left(y_{j+1}\right)}{y_{j+2}-y_{j+1}}-\frac{f\left(y_{j+1}\right)-f\left(y_{j}\right)}{y_{j+1}-y_{j}}\right\|}{y_{j+2}-y_{j}}\right)\left(y_{j+2}-y_{j}\right) \\
& =\varrho_{\Phi}^{2}(f ;[a, b], \xi \cup \eta) \leq V_{\Phi}^{2, R}(f ;[a, b], X) .
\end{aligned}
$$

Therefore

$$
V_{\Phi}^{2, R}(f ;[a, c])+V_{\Phi}^{2, R}(f ;[c, b]) \leq V_{\Phi}^{2, R}(f ;[a, b], X) .
$$

Remark 2.12. Inequality (2.5) cannot be replaced by an equality as can be readily verified by considering the example (2.9) with, for instance, $a=-2 / 3, b=2 / 3$, $c=0$. In this case, by considering the partition $c \xi=\{-2 / 3,-1 / 3,1 / 3,2 / 3\} 0$., one shows that

$$
\begin{aligned}
V_{\Phi}^{2, R}(f ;[-2 / 3,0])+V_{\Phi}^{2, R}(f ;[0,2 / 3])=0 \\
<2 \Phi(1) \\
\leq V_{\Phi}^{2, R}(f ;[-2 / 3,2 / 3], X) .
\end{aligned}
$$

\section{Main Results}

Now, we are ready to characterize the class of functions in $X^{[a, b]}$ of second bounded $\Phi$-variation in terms of the class of functions of (first) bounded $\Phi$-variation.

Theorem 3.1. Suppose $f \in B V_{\Phi}^{R}([a, b], X)$ and $F(x):=\int_{a}^{x} f(t) \mathrm{d} t$. Then $F \in B V_{\Phi}^{2, R}([a, b])$ and

$$
V_{\Phi}^{2, R}(F ;[a, b], X) \leq V_{\Phi}^{R}(f ;[a, b], X) .
$$

Proof. For a given partition $\xi \in \pi_{3}[a, b]$, putting $u:=\frac{t-x_{j+1}}{x_{j+2}-x_{j+1}}, v:=\frac{t-x_{j}}{x_{j+1}-x_{j}}$ and making use of Jensen inequality, we obtain

$$
\begin{aligned}
\varrho_{\Phi}^{2}(F ;[a, b], \xi) & =\sum_{j=0}^{m-2} \Phi\left(\frac{\left.\left\|\int_{0}^{1} f\left(u\left[x_{j+2}-x_{j+1}\right]+x_{j+1}\right) \mathrm{d} u-\int_{0}^{1} f\left(v\left[x_{j+1}-x_{j}\right]+x_{j}\right) \mathrm{d} v\right\|\right)}{x_{j+2}-x_{j}}\right)\left(x_{j+2}-x_{j}\right) \\
& =\sum_{j=0}^{m-2} \Phi\left(\frac{\left.\left\|\int_{0}^{1}\left\{f\left(u\left[x_{j+2}-x_{j+1}\right]+x_{j+1}\right)-f\left(u\left[x_{j+1}-x_{j}\right]+x_{j}\right)\right\} \mathrm{d} u\right\|\right)}{x_{j+2}-x_{j}}\right)\left(x_{j+2}-x_{j}\right) \\
& \leq \int_{0}^{1} \sum_{j=0}^{m-2} \Phi\left(\frac{\left\|f\left(u\left[x_{j+2}-x_{j+1}\right]+x_{j+1}\right)-f\left(u\left[x_{j+1}-x_{j}\right]+x_{j}\right)\right\|}{x_{j+2}-x_{j}}\right)\left(x_{j+2}-x_{j}\right) \mathrm{d} u
\end{aligned}
$$

As $u \in[0,1]$,

$$
\left[x_{j+2}-x_{j+1}\right]+x_{j+1}-u\left[x_{j+1}-x_{j}\right]-x_{j} \leq x_{j+2}-x_{j} .
$$

Thus, do $K=u\left[x_{j+2}-x_{j+1}\right]+x_{j+1}-u\left[x_{j+1}-x_{j}\right]-x_{j}$, we must 


$$
\begin{aligned}
\varrho_{\Phi}^{2}(F ;[a, b], \xi) & \leq \int_{0}^{1} \sum_{j=0}^{m-2} \Phi\left(\frac{\left\|f\left(u\left[x_{j+2}-x_{j+1}\right]+x_{j+1}\right)-f\left(u\left[x_{j+1}-x_{j}\right]+x_{j}\right)\right\|}{x_{j+2}-x_{j}}\right)\left(x_{j+2}-x_{j}\right) \mathrm{d} u \\
& =\int_{0}^{1} \sum_{j=0}^{m-2} \Phi\left(\frac{\left\|f\left(u\left[x_{j+2}-x_{j+1}\right]+x_{j+1}\right)-f\left(u\left[x_{j+1}-x_{j}\right]+x_{j}\right)\right\|}{K} \frac{K}{x_{j+2}-x_{j}}\right)\left(x_{j+2}-x_{j}\right) \mathrm{d} u \\
& \leq \int_{0}^{1} \sum_{j=0}^{m-2} \Phi\left(\frac{\left\|f\left(u\left[x_{j+2}-x_{j+1}\right]+x_{j+1}\right)-f\left(u\left[x_{j+1}-x_{j}\right]+x_{j}\right)\right\|}{K}\right) \frac{K}{x_{j+2}-x_{j}}\left(x_{j+2}-x_{j}\right) \mathrm{d} u \\
& \leq \int_{0}^{1} V_{\Phi}(f ;[a, b]) \mathrm{d} u=V_{\Phi}(f ;[a, b]) .
\end{aligned}
$$

Since this inequality holds for any partition $\xi \in \pi_{3}[a, b]$, we conclude that $\varrho_{\Phi}^{2}(F ;[a, b]) \leq V_{\Phi}(f ;[a, b])$, which implies (3.1).

Thus, replacing $V_{\Phi}^{R}(f,[a, b])$ by $\int_{a}^{b} \Phi\left(\left\|f^{\prime}(t)\right\|\right) \mathrm{d} t$ in (3.1) (cf. Proposition [11]), one obtains the following.

Corollary 3.2. Suppose $f \in B V_{\Phi}^{R}([a, b], X)$ and let $F(x):=\int_{a}^{x} f(t) \mathrm{d} t$. Then $F(x):=\int_{a}^{x} f(t) \mathrm{d} t$. and

$$
V_{\Phi}^{2, R}(F ;[a, b], X) \leq \int_{a}^{b} \Phi\left(\left\|f^{\prime}(t)\right\|\right) \mathrm{d} t .
$$

The following example shows that it is possible to have strict inequality in (3.2).

Example 3.3. Let $X=\mathbb{R}$ with the norm given by the absolute value. Let $F(x):=x^{2}$ defined on $[0,1]$. Suppose $\xi=\left\{x_{i}\right\}_{i=0}^{m} \in \pi_{3}[0,1]$. Then

$$
\begin{aligned}
\varrho_{\Phi}^{2}(f ;[a, b], \xi) & \left.=\sum_{j=0}^{m-2} \Phi\left(\mid \frac{\mid \frac{x_{j+2}^{2}-x_{j+1}^{2}}{x_{j+2}-x_{j+1}}-\frac{x_{j+1}^{2}-x_{j}^{2}}{x_{j+1}-x_{j}}}{x_{j+2}-x_{j}}\right)\right)\left(x_{j+2}-x_{j}\right)=\sum_{j=0}^{m-2} \Phi\left(\frac{x_{j+2}+x_{j+1}-x_{j+1}-x_{j}}{x_{j+2}-x_{j}}\right)\left(x_{j+2}-x_{j}\right) \\
& =\sum_{j=0}^{m-2} \Phi(1)\left(x_{j+2}-x_{j}\right)=\Phi(1)\left[\sum_{j=0}^{m-2}\left(x_{j+2}-x_{j+1}\right)+\sum_{j=0}^{m-2}\left(x_{j+1}-x_{j}\right)\right] \\
& =\Phi(1)\left[x_{m}-x_{1}+x_{m-1}-x_{0}\right]=\Phi(1)\left[1-x_{1}+x_{m-1}-0\right] .
\end{aligned}
$$

Taking now the supremum over all partitions $\xi \in \pi_{3}[0,1]$, one obtains

$$
\begin{gathered}
\sup _{\xi \in \pi_{3}[a, b]} \sum_{j=0}^{m-1} \Phi\left(\frac{\frac{f\left(x_{j+2}\right)-f\left(x_{j+1}\right)}{x_{j+2}-x_{j+1}}-\frac{f\left(x_{j+1}\right)-f\left(x_{j}\right)}{x_{j+1}-x_{j}}}{x_{j+2}-x_{j}}\right)\left(x_{j+2}-x_{j}\right) \\
=\sup _{\xi \in \pi_{3}[a, b]} \Phi(1)\left[1-x_{1}+x_{m-1}\right]=\Phi(1) 2 .
\end{gathered}
$$

Clearly, $F(x)=\int_{0}^{x} f(t) \mathrm{d} t$ (where $f(t)=2 t$ ) is absolutely continuous, $f^{\prime}(t)=2 \in L_{\Phi}[0,1]$, and

$$
\left\|f^{\prime}\right\|_{L_{\Phi}[0,1]}=\int_{0}^{1} \Phi\left(\left|f^{\prime}(\tau)\right|\right) \mathrm{d} \tau=\int_{0}^{1} \Phi(2) \mathrm{d} \tau=\Phi(2) .
$$

Now if, for instance $\Phi(t):=|t|^{p}$, for $1<p<\infty$, we have

$$
\left\|f^{\prime}\right\|_{L_{\Phi}[0,1]}=\Phi(2)=2^{p}>2=\Phi(1) 2=2 V_{\Phi}^{2, R}(f,[a, b]) .
$$

So, in all of these cases, inequality (3.2) is strict.

Lemma 3.4. Let $\Phi \in \mathcal{N}_{\infty}, E$ be a dense subset of $[a, b]$, and let $g: E \rightarrow X$ be a map, where $X$ is a Banach space, and let $K$ be a positive constant such that

$$
\sum_{j=0}^{m-1} \Phi\left(\frac{\left\|g\left(x_{j+1}\right)-g\left(x_{j}\right)\right\|}{x_{j+1}-x_{j}}\right)\left(x_{j+1}-x_{j}\right) \leq K,
$$

where $a \leq x_{0}<x_{1}<\cdots<x_{m-1}<x_{m} \leq b$ and $x_{j} \in E$ for $j=0,1,2, \cdots, m$. Then, $g_{E}(x-0)$ exists for all $x \in(a, b] \backslash E$, where $g_{E}(x-0)=\lim _{\substack{h \rightarrow 0^{+} \\ x-h \in E}} g(x-h)$.

An analogous assertion holds for $g_{E}(x+0)$, 
$(x \in[a, b))$ which is similarly defined.

Proof. It suffices to show that $g_{E}(x-0)$ exists for all $x \in(a, b] \backslash E$. Suppose that such is not the case; i.e., suppose that there is a $t \in(a, b] \backslash E$ such that $\lim _{\substack{h \rightarrow 0^{+} \\ t-h \in E}} g(t-h)=t-h \lim _{\substack{s \rightarrow t^{-} \\ s \in E}} g(s)$ does not exist.

Then by Cauchy's criterion (in the complete metric space $X$ ), there is an $\epsilon>0$ and an increasing sequence: $\left\{y_{n}\right\}_{n \geq 1} \in E \cap(a, t)$ such that $y_{n} \rightarrow t$ and

$$
\left\|g\left(y_{j+1}\right)-g\left(y_{j}\right)\right\|>\epsilon \text { for all } j .
$$

Since $y_{n} \rightarrow t$ we can find $N \in \mathbb{N}$ so that

$$
n \geq N \Rightarrow\left|y_{n}-t\right|<\frac{1}{2} .
$$

Thus, if $n \geq N$ we must

$$
y_{n+1}-y_{n} \leq\left|y_{n+1}-t\right|+\left|y_{n}-t\right|<1 \text {. }
$$

$$
e_{x_{0}}=\left\{j=0, \cdots, n-2: \frac{\left\|U\left(f ; x_{j+1}, x_{j+2}\right)-U\left(f ; x_{j}, x_{j+1}\right)\right\|}{x_{j+2}-x_{j}}>x_{0}\right\},
$$

then

$$
\begin{aligned}
\sum_{j=0}^{m-2}\left\|U\left(f ; x_{j+1}, x_{j+2}\right)-U\left(f ; x_{j}, x_{j+1}\right)\right\| & =\sum_{j=0}^{m-2} \frac{\left\|U\left(f ; x_{j+1}, x_{j+2}\right)-U\left(f ; x_{j}, x_{j+1}\right)\right\|}{x_{j+2}-x_{j}}\left(x_{j+2}-x_{j}\right) \\
& =\left(\sum_{j \notin e_{x_{0}}}+\sum_{j \in e_{x_{0}}}\right)\left(\frac{\left\|U\left(f ; x_{j+1}, x_{j+2}\right)-U\left(f ; x_{j}, x_{j+1}\right)\right\|}{x_{j+2}-x_{j}}\right)\left(x_{j+2}-x_{j}\right) \\
& \leq \sum_{j \notin e_{x_{0}}} x_{0}\left(x_{j+2}-x_{j}\right)+\sum_{j \in e_{x_{0}}}\left\|U\left(f ; x_{j+1}, x_{j+2}\right)-U\left(f ; x_{j}, x_{j+1}\right)\right\| .
\end{aligned}
$$

Now, for each $j \in e_{x_{0}}$ must be

$$
\left\|U\left(f ; x_{j+1}, x_{j+2}\right)-U\left(f ; x_{j}, x_{j+1}\right)\right\|<\frac{1}{r} \Phi\left(\frac{\left\|U\left(f ; x_{j+1}, x_{j+2}\right)-U\left(f ; x_{j}, x_{j+1}\right)\right\|}{x_{j+2}-x_{j}}\right)\left(x_{j+2}-x_{j}\right) .
$$

Substituting this in (3.4) we get

$$
\begin{aligned}
\sum_{j=0}^{m-2}\left\|U\left(f ; x_{j+1}, x_{j+2}\right)-U\left(f ; x_{j}, x_{j+1}\right)\right\| & \leq \sum_{j \notin x_{x_{0}}} x_{0}\left(x_{j+2}-x_{j}\right)+\frac{1}{r} \sum_{j \in e_{x_{0}}} \Phi\left(\frac{\left\|U\left(f ; x_{j+1}, x_{j+2}\right)-U\left(f ; x_{j}, x_{j+1}\right)\right\|}{x_{j+2}-x_{j}}\right)\left(x_{j+2}-x_{j}\right) \\
& =x_{0}(b-a)+\frac{1}{r} \sum_{j=0}^{n} \Phi\left(\frac{\left\|U\left(f ; x_{j+1}, x_{j+2}\right)-U\left(f ; x_{j}, x_{j+1}\right)\right\|}{x_{j+2}-x_{j}}\right)\left(x_{j+2}-x_{j}\right) .
\end{aligned}
$$

Since this inequality holds for every partition $\xi$ of $[a, b]$ we obtain

$$
V_{\Phi}^{2}(f ;[a, b], X) \leq x_{0}(b-a)+\frac{1}{r} V_{\Phi}^{2, R}(f ;[a, b], X) .
$$

Corollary 3.6. Suppose $X$ is a reflexive Banach space and let $\Phi \in \mathcal{N}_{\infty}$. If $V_{\Phi}^{2, R}(f ;[a, b], X)<\infty$ then $f$ is absolutely continuous on $[a, b]$. Moreover, by Theorem 2.6, $f^{\prime}$ is also absolutely continuous. 
Theorem 3.7. Let $X$ be a reflexive Banach space. If $F \in B V_{\Phi}^{2, R}([a, b], X)$ and $\Phi \in \mathcal{N}_{\infty}$ then there is a function $f \in B V_{\Phi}^{R}([a, b])$ such that $F^{\prime}=f$, Lebesgue-a.e., and

$$
V_{\Phi}^{2, R}(F ;[a, b], X) \leq V_{\Phi}^{R}(f ;[a, b], X) .
$$

Proof. We use the fact that $F$ is absolutely continuous and consequently it has strong derivative almost everywhere (Lebesgue) on $[a, b]$. Thus (see [12])

$$
\begin{aligned}
F(x) & =F(a)+\int_{a}^{x} F^{\prime}(t) \mathrm{d} t \\
& =F(a)+\int_{a}^{x} f(t) \mathrm{d} t .
\end{aligned}
$$

Therefore, by Theorem 3.1, the function $\varphi(x):=F(x)-F(a)$ is also of bounded second variation, in the sense of Riesz, and

$$
\begin{aligned}
V_{\Phi}^{2, R}(F ;[a, b], X) & =V_{\Phi}^{2, R}(F-F(a) ;[a, b], X) \\
& \leq V_{\Phi}^{R}(f,[a, b]) .
\end{aligned}
$$

Theorem 3.8. Let $X$ be a reflexive Banach space and $\Phi \in \Delta_{2}$, with constants $K>0$ and $t_{0}$ as in (2.2). If $F \in B V_{\Phi}^{2, R}([a, b], X)$ then there is a function $f \in B V_{\Phi}^{R}([a, b])$ such that $F^{\prime}=f$, Lebesgue-a.e., and $V_{\Phi}^{R}(f ;[a, b], X) \leq \frac{K}{2} V_{\Phi}^{2, R}(F ;[a, b], X)+2 \Phi\left(t_{0}\right)(b-a)$.

Proof. Since $F$ is absolutely continuous (Lemma 2.8) and $X$ is a reflexive Banach space, Theorem 2.6 ensures that $f$ is strongly differentiable a.e., with derivative strongly measurable. Let $E$ be a set of zero Lebesgue measure such that $F^{\prime}$ exists at every point of $\mathbb{E}:=[a, b] \backslash E$. Consider points $\left(x_{j}\right)_{0}^{m}$ in $[a, b] \cap \mathbb{E}^{\prime}$ such that $a \leq x_{0}<x_{1}<\cdots<x_{m} \leq b$, and let $h_{0}, h_{1}, \cdots, h_{m}$ be positive real numbers such that

$x_{-} j+h_{-} j<x_{-}\{j+1\}$ for all $j=0, \cdots, m-1$ and $x_{m-1}+h_{m-1}<x_{m}-h_{m}$.

Next, define a partition $\xi:=\left\{y_{j}\right\}_{j=0}^{2 m+1}$, of $[a, b]$ as

$$
y_{j}=\left\{\begin{array}{lll}
x_{j / 2} & \text { If } & 0 \leq j \leq 2 m-1 \text { is even, } \\
x_{j-1}+h_{\frac{j-1}{2}} & \text { If } & 1 \leq j \leq 2 m-1 \text { is odd } \\
x_{m}-h_{m} & \text { If } \quad j=2 m \\
x_{m} & \text { If } \quad j=2 m+1
\end{array}\right.
$$

Then

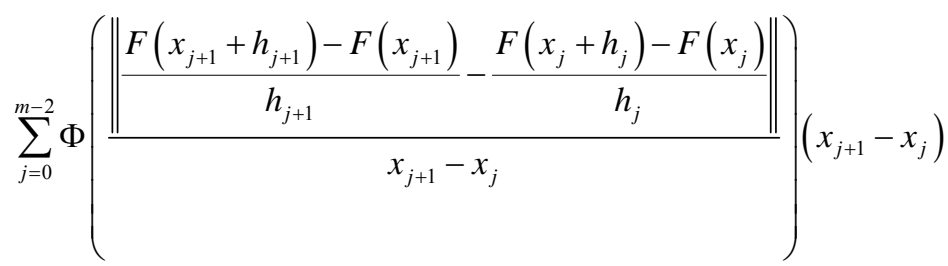

$+\Phi\left(\frac{\left\|\frac{F\left(x_{m}\right)-F\left(x_{m}-h_{m}\right)}{h_{m}}-\frac{F\left(x_{m-1}+h_{m-1}\right)-F\left(x_{m-1}\right)}{h_{m-1}}\right\|}{x_{m}-x_{m-1}}\right)\left(x_{m}-x_{m-1}\right)$

$=\sum_{j=0}^{m-2} \Phi\left(\frac{\| \frac{F\left(x_{j+1}+h_{j+1}\right)-F\left(x_{j+1}\right)}{h_{j+1}}-\frac{F\left(x_{j+1}\right)-F\left(x_{j}+h_{j}\right)}{x_{j+1}-x_{j}-h_{j}}}{x_{j+1}-x_{j}}+\frac{\frac{F\left(x_{j+1}\right)-F\left(x_{j}+h_{j}\right)}{x_{j+1}-x_{j}-h_{j}}-\frac{F\left(x_{j}+h_{j}\right)-F\left(x_{j}\right)}{h_{j}}}{x_{j+1}-x_{j}} \|\right)\left(x_{j+1}-x_{j}\right)$

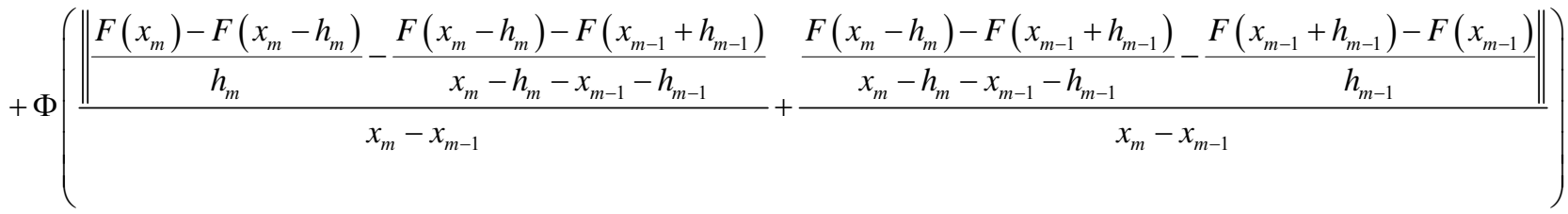

$\cdot\left(x_{m}-x_{m-1}\right)$,

using the convexity of $\Phi$ we obtain, 


$$
\begin{aligned}
& \leq \sum_{j=0}^{m-2} \frac{1}{2} \Phi\left(2 \frac{\left\|\frac{F\left(x_{j+1}+h_{j+1}\right)-F\left(x_{j+1}\right)}{h_{j+1}}-\frac{F\left(x_{j+1}\right)-F\left(x_{j}+h_{j}\right)}{x_{j+1}-x_{j}-h_{j}}\right\|}{x_{j+1}-x_{j}}\right)\left(x_{j+1}-x_{j}\right)
\end{aligned}
$$

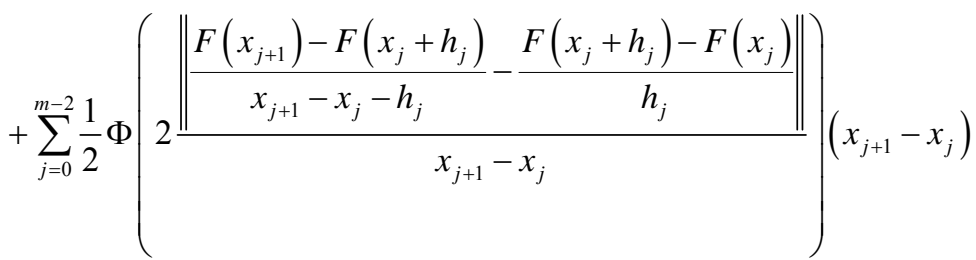

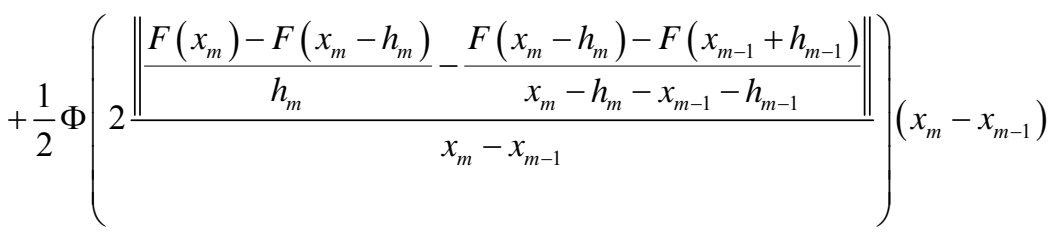

$$
\begin{aligned}
& +\frac{1}{2} \Phi\left(2 \frac{\left\|\frac{F\left(x_{m}-h_{m}\right)-F\left(x_{m-1}+h_{m-1}\right)}{x_{m}-h_{m}-x_{m-1}-h_{m-1}}-\frac{F\left(x_{m-1}+h_{m-1}\right)-F\left(x_{m-1}\right)}{h_{m-1}}\right\|}{x_{m}-x_{m-1}}\right)\left(x_{m}-x_{m-1}\right) \text {. }
\end{aligned}
$$

Let

$$
\begin{aligned}
& A_{1}=\left\{j=0,1, \cdots, m-2: \frac{\left\|\frac{F\left(x_{j+1}+h_{j+1}\right)-F\left(x_{j+1}\right)}{h_{j+1}}-\frac{F\left(x_{j+1}\right)-F\left(x_{j}+h_{j}\right)}{x_{j+1}-x_{j}-h_{j}}\right\|}{x_{j+1}-x_{j}} \geq \frac{t_{0}}{2}\right\} \\
& A_{2}=\left\{j=0,1, \ldots, m-2: \frac{\left\|\frac{F\left(x_{j+1}\right)-F\left(x_{j}+h_{j}\right)}{x_{j+1}-x_{j}-h_{j}}-\frac{F\left(x_{j}+h_{j}\right)-F\left(x_{j}\right)}{h_{j}}\right\|}{x_{j+1}-x_{j}} \geq \frac{t_{0}}{2}\right\} .
\end{aligned}
$$

Then, from the above inequality we obtain

$$
\begin{aligned}
& \sum_{j=0}^{m-2} \Phi\left(\frac{\left\|\frac{F\left(x_{j+1}+h_{j+1}\right)-F\left(x_{j+1}\right)}{h_{j+1}}-\frac{F\left(x_{j}+h_{j}\right)-F\left(x_{j}\right)}{h_{j}}\right\|}{x_{j+1}-x_{j}}\right)\left(x_{j+1}-x_{j}\right) \\
& +\Phi\left(\frac{\left.\left\|\frac{F\left(x_{m}\right)-F\left(x_{m}-h_{m}\right)}{h_{m}}-\frac{F\left(x_{m-1}+h_{m-1}\right)-F\left(x_{m-1}\right)}{h_{m-1}}\right\|\right)}{x_{m}-x_{m-1}}\right)\left(x_{m}-x_{m-1}\right)
\end{aligned}
$$




$$
\begin{aligned}
& \leq\left(\sum_{j \in A_{1}}+\sum_{j} \notin A_{1}\right) \frac{1}{2} \Phi\left(2 \frac{\| \frac{F\left(x_{j+1}+h_{j+1}\right)-F\left(x_{j+1}\right)}{h_{j+1}}-\frac{F\left(x_{j+1}\right)-F\left(x_{j}+h_{j}\right)}{x_{j+1}-x_{j}-h_{j}}}{x_{j+1}-x_{j}}\right)\left(x_{j+1}-x_{j}\right) \\
& +\left(\sum_{j \in A_{2}}+\sum_{j} \notin A_{2}\right) \frac{1}{2} \Phi\left(2 \frac{\| \frac{\frac{}{\left.x_{j+1}\right)-F\left(x_{j}+h_{j}\right)}}{x_{j+1}-x_{j}-h_{j}}-\frac{F\left(x_{j}+h_{j}\right)-F\left(x_{j}\right)}{h_{j}}}{x_{j+1}-x_{j}} \|\left(x_{j+1}-x_{j}\right)\right.
\end{aligned}
$$

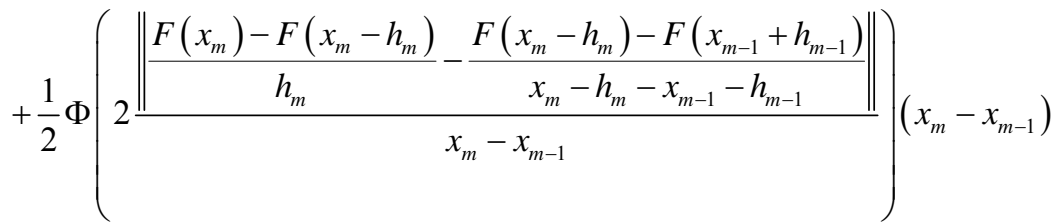

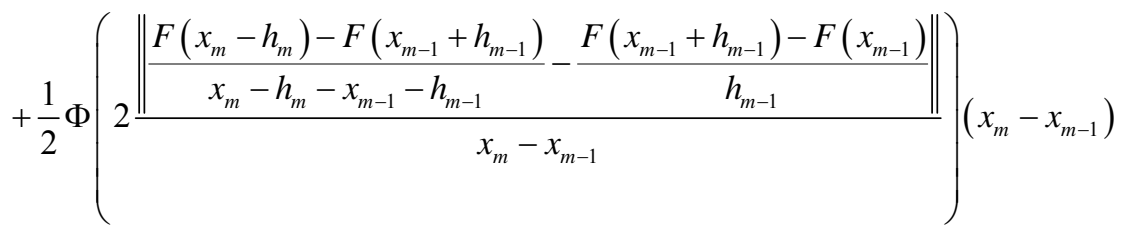

$$
\begin{aligned}
& \leq \sum_{j=0}^{m-2} \frac{K}{2} \Phi\left(\frac{\left\|\frac{F\left(x_{j+1}+h_{j+1}\right)-F\left(x_{j+1}\right)}{h_{j+1}}-\frac{F\left(x_{j+1}\right)-F\left(x_{j}+h_{j}\right)}{x_{j+1}-x_{j}-h_{j}}\right\|}{x_{j+1}-x_{j}}\right)\left(x_{j+1}-x_{j}\right) \\
& +\sum_{j=0}^{m-2} \frac{1}{2} \Phi\left(t_{0}\right)\left(x_{j+1}-x_{j}\right)+\sum_{j=0}^{m-2} \frac{K}{2} \Phi\left(\frac{\| \frac{F\left(x_{j+1}\right)-F\left(x_{j}+h_{j}\right)}{x_{j+1}-x_{j}-h_{j}}-\frac{F\left(x_{j}+h_{j}\right)-F\left(x_{j}\right)}{h_{j}}}{x_{j+1}-x_{j}}\right)\left(x_{j+1}-x_{j}\right) \\
& +\sum_{j=0}^{m-2} \frac{1}{2} \Phi\left(t_{0}\right)\left(x_{j+1}-x_{j}\right)+\frac{K}{2} \Phi\left(\frac{\left\|\frac{F\left(x_{m}\right)-F\left(x_{m}-h_{m}\right)}{h_{m}}-\frac{F\left(x_{m}-h_{m}\right)-F\left(x_{m-1}+h_{m-1}\right)}{x_{m}-h_{m}-x_{m-1}-h_{m-1}}\right\|}{x_{m}-x_{m-1}}\right)\left(x_{m}-x_{m-1}\right) \\
& +\frac{1}{2} \Phi\left(t_{0}\right)\left(x_{m}-x_{m-1}\right)+\frac{1}{2} \Phi\left(t_{0}\right)\left(x_{m}-x_{m-1}\right) . \\
& +\frac{K}{2} \Phi\left(\frac{\left\|\frac{F\left(x_{m}-h_{m}\right)-F\left(x_{m-1}+h_{m-1}\right)}{x_{m}-h_{m}-x_{m-1}-h_{m-1}}-\frac{F\left(x_{m-1}+h_{m-1}\right)-F\left(x_{m-1}\right)}{h_{m-1}}\right\|}{x_{m}-x_{m-1}}\right)\left(x_{m}-x_{m-1}\right)
\end{aligned}
$$


Thus,

$$
\sum_{j=0}^{m-2} \Phi\left(\frac{\left\|\frac{F\left(x_{j+1}+h_{j+1}\right)-F\left(x_{j+1}\right)}{h_{j+1}}-\frac{F\left(x_{j}+h_{j}\right)-F\left(x_{j}\right)}{h_{j}}\right\|}{x_{j+1}-x_{j}}\right)\left(x_{j+1}-x_{j}\right) \leq \frac{K}{2} V_{\Phi}^{2, R}(F ;[a, b], X)+2 \Phi\left(t_{0}\right)(b-a)
$$

Making $h_{j} \rightarrow 0, \quad j=0,1,2, \cdots, m$, one gets

$$
\sum_{j=0}^{m-1} \Phi\left(\frac{\left\|F^{\prime}\left(x_{j+1}\right)-F^{\prime}\left(x_{j}\right)\right\|}{x_{j+1}-x_{j}}\right)\left(x_{j+1}-x_{j}\right) \leq \frac{K}{2} V_{\Phi}^{2, R}(F ;[a, b], X)+2 \Phi\left(t_{0}\right)(b-a)
$$

therefore, by Lemma $3.4 F_{\mathbb{E}}^{\prime}(x-0)$ and $F_{\mathbb{E}}^{\prime}(x+0)$ exists for all $x \in(a, b] \backslash \mathbb{E}$. Define now the function

$$
f(x):=\left\{\begin{array}{ccc}
F^{\prime}(x) & \text { when } & x \in \mathbb{E}, \\
F_{\mathbb{E}}^{\prime}(x-0) & \text { when } & x \in(a, b] \backslash \mathbb{E}, \\
F_{\mathbb{E}}^{\prime}(a+0) & \text { if } & x=a \text { and } a \notin \mathbb{E} .
\end{array}\right.
$$

Clearly, $F^{\prime}=f$ almost everywhere. We need just to verify that $f$ satisfies (3.5). Let $\xi:=\left\{x_{j}\right\}_{j=0}^{m}$ be a parti-

$$
\begin{aligned}
& \sum_{j=0}^{k-2} \Phi\left(\frac{\left\|f\left(x_{j+1}\right)-f\left(x_{j}\right)\right\|}{x_{j+1}-x_{j}}\right)\left(x_{j+1}-x_{j}\right)+\Phi\left(\frac{\left\|f\left(x_{k^{\prime}}\right)-f\left(x_{k-1}\right)\right\|}{x_{k^{\prime}}-x_{k-1}}\right)\left(x_{k^{\prime}}-x_{k-1}\right) \\
& +\Phi\left(\frac{\left\|f\left(x_{k+1}\right)-f\left(x_{k^{\prime}}\right)\right\|}{x_{k+1}-x_{k^{\prime}}}\right)\left(x_{k+1}-x_{k^{\prime}}\right)+\sum_{j=k+1}^{m-2} \Phi\left(\frac{\left\|f\left(x_{j+1}\right)-f\left(x_{j}\right)\right\|}{x_{j+1}-x_{j}}\right)\left(x_{j+1}-x_{j}\right) \\
& =\sum_{j=0}^{k-2} \Phi\left(\frac{\left\|F^{\prime}\left(x_{j+1}\right)-F^{\prime}\left(x_{j}\right)\right\|}{x_{j+1}-x_{j}}\right)\left(x_{j+1}-x_{j}\right)+\Phi\left(\frac{\left\|F^{\prime}\left(x_{k^{\prime}}\right)-F^{\prime}\left(x_{k-1}\right)\right\|}{x_{k^{\prime}}-x_{k-1}}\right)\left(x_{k^{\prime}}-x_{k-1}\right) \\
& +\Phi\left(\frac{\left\|F^{\prime}\left(x_{k+1}\right)-F^{\prime}\left(x_{k^{\prime}}\right)\right\|}{x_{k+1}-x_{k^{\prime}}}\right)\left(x_{k+1}-x_{k^{\prime}}\right)+\sum_{j=k+1}^{m-2} \Phi\left(\frac{\left\|F^{\prime}\left(x_{j+1}\right)-F^{\prime}\left(x_{j}\right)\right\|}{x_{j+1}-x_{j}}\right)\left(x_{j+1}-x_{j}\right) \\
& \leq \frac{K}{2} V_{\Phi}^{2, R}(F ;[a, b], X)+2 \Phi\left(t_{0}\right)(b-a)
\end{aligned}
$$

and taking the limit as $x_{k^{\prime}} \rightarrow x_{k}$ we have

$$
\begin{aligned}
& \sum_{j=0}^{k-2} \Phi\left(\frac{\left\|f\left(x_{j+1}\right)-f\left(x_{j}\right)\right\|}{x_{j+1}-x_{j}}\right)\left(x_{j+1}-x_{j}\right)+\Phi\left(\frac{\left\|f\left(x_{k}\right)-f\left(x_{k-1}\right)\right\|}{x_{k}-x_{k-1}}\right)\left(x_{k}-x_{k-1}\right) \\
& +\Phi\left(\frac{\left\|f\left(x_{k+1}\right)-f\left(x_{k}\right)\right\|}{x_{k+1}-x_{k^{\prime}}}\right)\left(x_{k+1}-x_{k}\right)+\sum_{j=k+1}^{m-2} \Phi\left(\frac{\left\|f\left(x_{j+1}\right)-f\left(x_{j}\right)\right\|}{x_{j+1}-x_{j}}\right)\left(x_{j+1}-x_{j}\right) \\
& \leq \frac{K}{2} V_{\Phi}^{2, R}(F ;[a, b], X)+2 \Phi\left(t_{0}\right)(b-a) .
\end{aligned}
$$


If, on the other hand, $x_{0}=a$ is the only point of $\xi$ not in $\mathbb{E}$, then we may consider a collection $\left\{x_{0^{\prime}}, x_{1}, \cdots, x_{k-1}, x_{k}, x_{k+1}, \cdots, x_{m}\right\}$, where $a<x_{0^{\prime}}<x_{1}$. Then

$$
\lim _{x_{0^{\prime}} \rightarrow x_{0}} f\left(x_{0^{\prime}}\right)=\lim _{x_{0^{\prime}} \rightarrow x_{0}} F^{\prime}\left(x_{0^{\prime}}\right)=F^{\prime}\left(x_{0}+0\right)=f(a) .
$$

Thus, proceeding as above, we get

$$
\Phi\left(\frac{\left\|f\left(x_{1}\right)-f\left(x_{0^{\prime}}\right)\right\|}{x_{1}-x_{0^{\prime}}}\right)\left(x_{1}-x_{0^{\prime}}\right)+\sum_{j=1}^{m-2} \Phi\left(\frac{\left\|f\left(x_{j+1}\right)-f\left(x_{j}\right)\right\|}{x_{j+1}-x_{j}}\right)\left(x_{j+1}-x_{j}\right) \leq \frac{K}{2} V_{\Phi}^{2, R}(F ;[a, b], X)+2 \Phi\left(t_{0}\right)(b-a) .
$$

and taking the limit as $x_{0^{\prime}} \rightarrow x_{0}$ :

$$
\Phi\left(\frac{\left\|f\left(x_{1}\right)-f\left(x_{0}\right)\right\|}{x_{1}-x_{0}}\right)\left(x_{1}-x_{0}\right)+\sum_{j=1}^{m-2} \Phi\left(\frac{\left\|f\left(x_{j+1}\right)-f\left(x_{j}\right)\right\|}{x_{j+1}-x_{j}}\right)\left(x_{j+1}-x_{j}\right) \leq \frac{K}{2} V_{\Phi}^{2, R}(F ;[a, b], X)+2 \Phi\left(t_{0}\right)(b-a) .
$$

To complete the proof that $f$ is of bounded (first) variation (in sense of Riesz), let $\xi:=\left\{x_{j}\right\}_{j=0}^{m} \in \pi_{3}([a, b])$ arbitrary, and set

$$
\xi^{\prime}=\left\{x_{0^{\prime}}, x_{1^{\prime}}, \cdots, x_{k-1}, x_{k^{\prime}}, x_{k+1}, \cdots, x_{m^{\prime}}\right\}
$$

where: $x_{j^{\prime}}=x_{j}$ if $x_{j} \in \mathbb{E}$; if $a \neq x_{j} \notin \mathbb{E}$. Then, $\underset{j-1}{x}<\underset{j^{\prime}}{x}<\underset{j}{x}$, where as if $a \notin \mathbb{E}$ take $a<x_{0^{\prime}}<x_{1^{\prime}}$.

Then, from the above estimations we obtain

$$
\begin{aligned}
& \sum_{j=0}^{m-2} \Phi\left(\frac{\left\|f\left(x_{j+1}\right)-f\left(x_{j}\right)\right\|}{x_{j+1}-x_{j}}\right)\left(x_{j+1}-x_{j}\right) \\
& \leq \frac{K}{2} V_{\Phi}^{2, R}(F ;[a, b], X)+2 \Phi\left(t_{0}\right)(b-a) .
\end{aligned}
$$

Hence, by taking the supreme over all partitions $\xi$ of $[a, b]$ :

$$
\begin{aligned}
V_{\Phi}^{R}(f,[a, b]) \leq & \frac{K}{2} V_{\Phi}^{2, R}(F ;[a, b], X) \\
& +2 \Phi\left(t_{0}\right)(b-a) .
\end{aligned}
$$

Corollary 3.9. Let $X$ be a reflexive Banach space and let $\Phi \in \Delta_{2}$ globally, with $\Delta_{2}$-constant $K>0$. If $F \in B V_{\Phi}^{2, R}([a, b], X)$ then there is a function $f \in B V_{\Phi}^{R}([a, b])$ such that $F^{\prime}=f$, Lebesgue-a.e., and

$$
\begin{aligned}
V_{\Phi}^{2, R}(F ;[a, b], X) & \leq \int_{a}^{b} \Phi\left(\left\|F^{\prime \prime}(t)\right\|\right) \mathrm{d} t \\
& \leq \frac{K}{2} V_{\Phi}^{2, R}(F ;[a, b], X) .
\end{aligned}
$$

Proof. Theorem 3.8 ensures the existence of a function $f \in B V_{\Phi}^{R}([a, b])$ (in the sense of Riesz) such that $F^{\prime}=f$, and

$$
\begin{aligned}
V_{\Phi}^{2, R}(F ;[a, b], X) & \leq V_{\Phi}^{R}(f ;[a, b], X) \\
& \leq \frac{K}{2} V_{\Phi}^{2, R}(F ;[a, b], X) .
\end{aligned}
$$

Now, by Theorem $2.6 f$ must be strongly differentiable a.e. on $[a, b]$ with derivative $f^{\prime}$ strongly measurable and Bochner integrable on $[a, b]$, and

$$
\begin{aligned}
& V_{\Phi}^{R}(f,[a, b])=\int_{a}^{b} \Phi\left(\left\|f^{\prime}(t)\right\|\right) \mathrm{d} t \text {. Thus } \\
& \quad V_{\Phi}^{2, R}(F ;[a, b], X) \\
& \leq \int_{a}^{b} \Phi\left(\left\|f^{\prime}(t)\right\|\right) \mathrm{d} t=\int_{a}^{b} \Phi\left(\left\|F^{\prime \prime}(t)\right\|\right) \mathrm{d} t \\
& \leq \frac{K}{2} V_{\Phi}^{2, R}(F ;[a, b], X)
\end{aligned}
$$

That Theorem 5.8 (Corollary 3.9) generalizes Riesz's lemma is brought forward by considering, for $p>1$, the function $\Phi_{p}(t):=|t|^{p}$, which has sharp $\Delta_{2}$-constant $K=2^{p}$. Indeed, in this case we have:

Corollary 3.10. Let $X$ be a reflexive Banach space. If $p>1$, and $F \in B V_{\Phi_{p}}^{2, R}([a, b], X)$ then there is a function $f \in B V_{\Phi_{p}}^{R}([a, b])$ such that $F^{\prime}=f$, Lebesgue-a.e., and

$$
\begin{aligned}
V_{\Phi_{p}}^{2, R}(F ;[a, b], X) & \leq \int_{a}^{b}\left\|F^{\prime \prime}(t)\right\|^{p} \mathrm{~d} t \\
& \leq 2^{p-1} V_{\Phi_{p}}^{2, R}(F ;[a, b], X) .
\end{aligned}
$$

\section{REFERENCES}

[1] Ch. J. de la Vallée Poussin, "Sur la Convergence des Formules d'Interpolation Entre Ordennées Equidistantes," Bulletin de la Classe des Sciences, No. 4, 1908, pp. 314-410.

[2] F. Riesz, "Sur Certains Systems Singuliers d'Equations Integrates," Annales Scientifiques de l'École Normale Supérieure, Vol. 3, No. 28, 1911, pp. 33-68.

[3] A. M. Russell and C. J. F. Upton, "A Generalization of a Theorem by F. Riesz," Mathematical Analysis, Vol. 9, No. 1, 1983, pp. 69-77. doi:10.1007/BF01903991

[4] N. Merentes, "On Functions of Bounded (p,2)-Variation," Collectanea Mathematica, Vol. 43, No. 2, 1992, pp. 117 123.

[5] N. Merentes and S. Rivas, "El Operador de Composici? n 
en Espacios de Funciones con Algún Tipo de Variación Acotada," IX Escuela Venezolana de Matemáticas, Facultad de Ciencias-ULA, Mérida, 1996.

[6] A. W. Roberts and D. E. Varberg, "Functions of Bounded Convexity," Bulletin of the AMS-American Mathematical Society, Vol. 75, No. 3, 1969, pp. 568-572. doi:10.1090/S0002-9904-1969-12244-5

[7] F. Szigeti, "Composition of Sobolev Functions and Applications," Notas de Matematicas, No. 86, 1987, pp. 125.

[8] L. Maligranda, "Orlicz Spaces and Interpolation," Semi- nars in Mathematics 5, University of Campinas, Campinas, 1989.

[9] J. Diestel and J. J. Uhl, "Vector Measures," Mathematical Surveys, Vol. 15, 1977.

[10] V. V. Chistyakov, "Mappings of Generalized Variation and Composition Operators," Journal of Mathematical Sciences, Vol. 110, No. 2, 2002, pp. 2455-2466. doi:10.1023/A:1015018310969

[11] V. Barbu and Th. Precupanu, "Convexity and Optimization on Banach Spaces," Sijthof and Noordhoff, Nederlands, 1978. 